\title{
ESCUELA Y VIOLENCIAS: DESPLEGANDO UNA RELACIÓN PROBLEMÁTICA
}

\author{
Pablo Francisco Di Leo (Universidad de Buenos Aires- \\ Consejo Nacional de Investigaciones Científicas y Técnicas)* \\ pfdileo@gmail.com
}

Recibido: 14/07/2011 Aceptado: 8/11/2011

\section{Resumen}

Durante los últimos años, las políticas públicas, los medios de comunicación masiva y los agentes escolares en Argentina vienen coincidiendo -aunque con sentidos muy diversos- en identificar a la violencia como uno de los problemas centrales de las instituciones educativas. En el artículo hago un recorrido en torno a los principales aportes del campo de las ciencias sociales para la problematización y análisis de las relaciones entre violencias y escuela, haciendo especial hincapié en recientes indagaciones realizadas en nuestro país. Organizo dicho recorrido a partir de tres grandes dimensiones que, si bien se encuentran íntimamente vinculadas entre sí, constituyen los ejes centrales desde los cuales la investigación social viene abordando las violencias escolares: condiciones histórico-sociales, factores institucionales y experiencias escolares. Alrededor de las dos últimas dimensiones, presento parte de los resultados de mi Tesis Doctoral en la que, a partir de datos construidos mediante diversas técnicas de investigación social de tipo cualitativo, identifico y analizo las relaciones entre los principales tipos de clima social escolar y los procesos de subjetivación en escuelas secundarias públicas de la Ciudad de Buenos Aires.

\section{Palabras clave}

Violencias - Escuela - Climas Sociales - Subjetivación - Juventudes.

* Doctor en Ciencias Sociales. Universidad de Buenos Aires. Magíster en Políticas Sociales. Universidad de Buenos Aires. Licenciado en Sociología. Universidad de Buenos Aires. 


\title{
SCHOOL AND VIOLENCES: DISPLAYING A PROBLEMATIC RELATIONSHIP
}

\begin{abstract}
In recent years, public policies, mass media and school agents in Argentina are agreeing -albeit with very different ways- to identify violence as one of the central problems of educational institutions. In this article I present the main contributions of social sciences to the problematizing and analysis of the relationship between violences and school, with special emphasis on recent investigations conducted in our country. The description is organized based on three large categories that are closely related and constitute the three central themes used for social investigation on school violence: socio-historical conditions, institutional factors and school experiences. Around the last two dimensions, I present part of the results of my PhD Thesis in which, based on data constructed by various techniques of qualitative social research, I identify and analyze the relationships between the main types of school social climates and the subjectivation processes in public high schools located in Buenos Aires City.
\end{abstract}

\section{Key words}

Violences - School - Social Climates - Subjectivation - Youths.

\section{Introducción}

A partir de estudios cualitativos y cuantitativos desarrollados recientemente por nuestro equipo de investigación en escuelas secundarias públicas y, fundamentalmente, a partir del trabajo de campo de mi Tesis Doctoral, encontramos que uno de los fenómenos institucionales percibidos por sus agentes como un problema central y creciente es la violencia. ${ }^{1}$ Tanto de los resultados de investigaciones sobre el tema desarrolladas en el país y en otros países de la región, como de nuestras indagaciones, surge que los climas sociales escolares funcionan como prismas que refractan de maneras particulares hacia el interior de la institución, las violencias provenientes del exterior. Las características de las relaciones interpersonales, el contexto en el que estas se desarrollan y las percepciones de los sujetos sobre las mismas tienen una alta influencia en las posibilidades de las instituciones educativas para potenciar, disminuir, prevenir y/o resolver las situaciones de violencia en su ámbito (Abramovay, 2006; Kornblit, 2008; Míguez, 2008; Di Leo, 2009; Noel, 2009).

Asimismo, los significados en torno a la violencia escolar atribuidos por sus agentes marcan un amplio abanico que va desde los actos directos de agresión física y/o verbal -que son los primeros que generan la alarma institucional- hasta diversas formas cotidianas de agresión atribuidas a las relaciones interpersonales -discriminación, falta de respeto, abuso o crisis de autoridad, falta de contención, entre otras. Sin embargo, como señala Bernard Charlot (2006), es preciso deconstruir la pseudo-evidencia del concepto. Cuando nos 
encontramos ante un problema social de definición, tenemos que preguntarnos quién está proponiendo qué definición, en cuál contexto, con qué objetivo e implicaciones personales y sociales. Como indica Pierre Bourdieu (2007), el poder de nominación se encuentra en el centro de las disputas por el poder simbólico. El debate que viene dándose desde hace más de diez años en todo el mundo acerca de lo que merece ser llamado violencia escolar constituye una lucha simbólica.

En nuestras sociedades dicha categoría se encuentra asociada a los comportamientos o palabras inaceptables, insoportables, contrarias a la civilización, la humanidad, la modernidad. Por ello, cada agente busca introducir en la lista de las violencias lo que vive como inaceptable en el comportamiento de los otros.

Como desarrollo en el artículo, desde los inicios de los sistemas educativos modernos se generó sobre la problemática de las violencias escolares una intensa lucha simbólica por su definición y, asociada a la misma, por su control. Durante las últimas décadas, las ciencias sociales vienen jugando roles estratégicos en dicho proceso de problematización y definición. Sin embargo, el principal aporte de dichos abordajes no surge de la delimitación conceptual del fenómeno, sino de la generación de nuevas dimensiones analíticas para abordar la problemática relación entre los sujetos y las instituciones educativas en el actual contexto de nuestras sociedades democráticas. Las violencias escolares pueden abordarse como analizadores de los (des)encuentros entre el programa institucional de la escuela clásica y los actuales procesos de subjetivación juvenil.

A continuación, propongo un recorrido sobre los principales aportes de las ciencias sociales para la problematización y análisis de los vínculos entre violencias y escuela, haciendo especial hincapié en recientes indagaciones desarrolladas en Argentina. Organizo dicho recorrido a partir de tres grandes dimensiones que, si bien se encuentran íntimamente relacionadas entre sí, constituyen los tres principales ejes desde los cuales la investigación social vienen abordando las violencias escolares: a) condiciones histórico-sociales, b) factores institucionales y c) experiencias escolares. Alrededor de las dos últimas dimensiones, presento parte de los resultados de mi Tesis Doctoral, en la que analizo los principales tipos de clima social presentes en escuelas secundarias públicas de la Ciudad Autónoma de Buenos Aires (CABA), reflexionando sobre sus vinculaciones con los procesos de subjetivación juvenil en nuestra sociedad actual.

\section{Condiciones histórico-sociales}

Durante la década de 1980, en los primeros años del retorno del régimen democrático en Argentina, gran parte de los debates e investigaciones en el campo educativo giraron en torno a la instalación de una convivencia demo- 
crática y participativa. Desde estas posiciones discursivas se consideraba que la escuela podría convertirse en una institución estratégica para revertir las estructuras autoritarias -tanto a nivel objetivo como subjetivo- construidas por años de regímenes dictatoriales (Gentili, 1994; Filmus, 2003).

Sin embargo, a fines de los años ' 80 , en el marco de las transformaciones estructurales iniciadas a mediados de la década anterior, las políticas educativas neoliberales y las características de la nueva condición juvenil, emergen los problemas de convivencia en las escuelas secundarias como uno de los principales síntomas de la crisis del proyecto institucional escolar (Dubet y Martuccelli, 1998; 2000; Dubet, 2006). Al mismo tiempo que se profundizan sus condiciones de precariedad y fragmentación material y simbólica, las instituciones escolares se enfrentan a una contradicción para la que no estaban preparadas: tienen el mandato de incluir a los sectores sociales a los cuales las políticas económico-sociales tienden a marginar. La imposibilidad de superar este dilema se convierte en uno de los principales condicionantes de la expansión y complejización de las situaciones de violencia escolar (Filmus, 2003; Corea y Lewkowicz, 2004).

En el campo académico, durante la última década, las investigaciones sobre las violencias en la escuela se multiplican y desplazan sus miradas desde perspectivas individuales -centradas en factores psicológicos y/o familiares de los agentes participantes- hacia enfoques socio-culturales. Dichos episodios comienzan a entenderse como fenómenos histórico-socialmente situados y la mayoría de las indagaciones sobre los mismos se dirigen a identificar sus articulaciones con el incremento de las desigualdades económico-sociales, culturales, de género y étnicas. La explicitación de dichas influencias contribuiría a desnaturalizar la doxa constitutiva del campo educativo (Filmus, 2003; Kaplan, 2006). ${ }^{2}$

Las premisas de este tipo de abordajes de las violencias escolares se encuentran, en los inicios de la conformación del campo sociológico, a principios del siglo XX, en la obra de Émile Durkheim, quien señala que "la educación, lejos de tener simplemente por fin desarrollar al hombre tal como sale de las manos de la naturaleza, tienen por objeto extraer de allí un hombre enteramente nuevo; crea un ser que no existe, salvo en el estado de germen indiscernible: el ser social" (Durkheim, 1998: 18).

Para lograr dicho objetivo, la relación pedagógica constitutiva de las instituciones escolares no sólo es asimétrica en relación a la edad, la experiencia o el saber, si no que es esencialmente antagónica. Por ende, para Durkheim (1972; 1998), la relación de desigualdad estructural es constitutiva de la escuela moderna. Esta concepción es retomada y profundizada desde los campos de la sociología y la pedagogía crítica desde la década de 1960. En Francia, sus principales referentes son Pierre Bourdieu y Jean-Claude Passeron (1981), mientras que, en América Latina, tiene su formulación clásica en la Pedagogía 
del oprimido de Paulo Freire (1997). Si bien responden a contextos políticos y académicos bien diferenciados, ambas obras contribuyen a establecer una ruptura epistemológica con dos de los principales postulados del programa institucional escolar moderno:

a) La escuela como igualadora social: en tensión con la utopía igualadora de la socialización escolar -cuya expresión clásica en Argentina fue el discurso sarmientino de la formación del ciudadano-, el sistema educativo contribuye, mediante el ejercicio de la violencia simbólica legítima estatal, a la reproducción de la desigual distribución de los diversos tipos de capital en el espacio social.

b) La autonomía de la cultura escolar: por ende, lejos de haber constituido un espacio independiente de los conflictos y violencias inherentes a la vida económico-política de las sociedades modernas, las relaciones pedagógicas participan del proceso de reproducción de sus estructuras de dominación y opresión. La violencia simbólica escolar tiene una productividad mayor que otros tipos de violencia, pues se ejerce precisamente en la medida en que sus agentes no la perciben como tal.

Por ende, para ambas corrientes de pensamiento crítico, resulta fundamental el trabajo de ruptura con la doxa y los habitus reproducidos por la relación pedagógica. Si bien la mirada sociológica de Bourdieu y Passeron (1981) busca captar los conflictos inherentes al campo educativo, tiene una postura pesimista respecto a sus posibilidades de cambio, considerando que el mismo no puede surgir desde la institución escolar, sino a partir de una profunda transformación del sistema educativo en su conjunto, utilizando como insumos centrales los análisis sociológicos dirigidos a la explicitación y, por ende, desnaturalización, de sus mecanismos de reproducción.

En esta corriente analítica, podemos citar las investigaciones sobre las diversas formas de segregación espacial y escolar, desarrolladas en Francia a mediados de los noventa por Eric Debarbieux (1996), Robert Ballion y Jean-Paul Payet (Charlot y Emin, 1997). De estos trabajos surge que la problemática de las violencias escolares se articula con la creciente fragmentación del sistema educativo, a partir de la cual las composiciones sociales y étnicas de las instituciones educativas presentan diferencias muy marcadas. Este fenómeno genera un círculo vicioso a partir del cual los padres de sectores medios tienden a evitar los establecimientos con altas tasas de estudiantes inmigrantes, reforzando los procesos de segregación escolar y social.

Según Payet (1997), este fenómeno se refuerza a partir de un doble movimiento: a) la segregación espacial de las escuelas dentro de barrios periféricos; b) la segregación al interior de las instituciones escolares mismas, que implica la distribución de los estudiantes en diferentes divisiones de un mismo nivel. De esta manera, en contextos de creciente exclusión estructural, la fragmentación, tanto del sistema educativo como de los climas sociales escolares-dimensión 
que retomamos luego-, contribuye a los procesos de descivilización: las heterocoacciones, el bajo control de las emociones, las agresiones, la inestabilidad emocional, etc., se convierten en síntomas de la expansión de un individualismo negativo, "un individualismo por falta de marcos y no por exceso de intereses subjetivos" (Castel, 1997, p. 472; Tenti Fanfani, 1999; Míguez, 2000).

De la mano de la hegemonía neoliberal, el progresivo retiro y/o crisis de las instituciones estatales disminuyen las posibilidades de acceso de los jóvenes, especialmente aquellos en situación de vulnerabilidad o desafiliación, a las herramientas simbólicas y materiales básicas para constituirse como sujetos plenos (Filmus y Miranda, 1999; Tenti Fanfani, 1999; Tiramonti y Montes, 2008; Tedesco, 2008). Para estos individuos, las instituciones han dejado de ser anclajes de proyección de un futuro y de construcción de identidades, de un orden simbólico integrador. En este marco entra en crisis el programa institucional de la escuela republicana, que se había estructurado en torno a la "constitución de un sujeto universal" (Duschatzky y Corea, 2002; Corea y Lewkowicz, 2004; Dubet, 2006). Una de las respuestas posibles a la exclusión, vivida como fracaso de las expectativas depositadas en la escolarización, puede ser el encapsulamiento en el propio grupo y el enfrentamiento -que muchas veces lleva a situaciones violentas- hacia lo considerado como el exo-grupo: la escuela y sus agentes (Kessler, 2004; Noel, 2009).

Durante las últimas décadas, a nivel internacional, la categoría de incivilidades viene siendo utilizada para analizar cómo impactan en las instituciones escolares estas nuevas condiciones socio-estructurales. Dicho concepto hace referencia a las violencias antisociales y antiescolares, de índole simbólico y, por lo tanto, opacas a la conciencia cotidiana: "La incivilidad es la revelación de un caos posible, una pérdida de sentido y de confianza sobre uno mismo y sobre los otros" (Charlot y Emin, 1997, p. 19).

En una investigación comparativa, mediante una encuesta realizada a una muestra de 14.000 estudiantes y 6.000 docentes y responsables pedagógicos, el equipo de Debarbieux construye datos que evidencian las articulaciones entre las violencias en ámbitos escolares con las condiciones sociodemográficas de sus poblaciones (Charlot y Emin, 1997). De su análisis surge que los establecimientos con mayor proporción de alumnos en situaciones socialmente vulnerables viven más frecuentemente delitos e infracciones, tiene climas sociales más degradados y un mayor predomino de sentimientos de inseguridad (Kaplan, 2006).

A partir del análisis de las vinculaciones entre las incivilidades y los contextos socio-económicos de las instituciones educativas, los sociólogos franceses comienzan a distinguir tres sentidos interrelacionados de las violencias escolares: 
- Violencia de la escuela: pone de relieve la violencia institucional, la dominación simbólica que la escuela ejerce como tal y se encuentra, por ende, en la génesis de los sistemas educativos modernos.

- Violencia hacia la escuela: es aquella que está dirigida hacia los agentes e infraestructura escolares y, en general, actúan como formas de contestación o reflejo frente a las violencias impuestas por la institución.

- Violencia en la escuela: hace referencia a la irrupción de la vida social en el seno de la vida escolar, a través de personas y/o formas delictivas provenientes del exterior de la institución educativa, especialmente conflictos entre bandas y narcotráfico (Charlot, 2002).

En Brasil, retomando los abordajes teórico-metodológicos desarrollados en Francia, se inicia en 2002 una serie de relevamientos dirigidos a caracterizar las violencias escolares a nivel nacional. En dicho año se crea el Observatorio de Violencias en las Escuelas, a partir de un acuerdo entre la Universidad Católica de Brasilia y UNESCO Brasil (Abramovay y Das Graças Rua, 2002). A partir de los datos construidos durante estos primeros años de investigación sociológica, surgen los siguientes núcleos de articulación entre dimensiones contextuales y violencias escolares:

- Incidencia de grupos criminales y del narcotráfico en la cotidianeidad escolar.

- Incidencia del incremento de la matrícula del nivel secundario, especialmente la escolarización masiva de sectores populares con condiciones de vida precarias.

- Incidencia de representaciones sociales xenofóbicas y/o discriminadoras entre agentes escolares.

- Incidencia de la crisis económica y los cambios del mercado de trabajo en la articulación entre la escuela y los proyectos de los sectores populares, que generan el debilitamiento de su eficacia simbólica y socializadora.

Aunque en nuestro país el campo de los estudios sociales de las violencias escolares es aún incipiente y fragmentario, pueden considerase como antecedentes los análisis centrados en las formas ocultas de discriminación escolar generadas por las representaciones docentes peyorativas hacia los alumnos provenientes de sectores sociales más vulnerables. Como también surge de mi investigación doctoral, para la mayoría de los docentes el aumento de las situaciones de violencia en el ámbito escolar tiene como una de sus causas principales el crecimiento de la cantidad de alumnos provenientes de familias y/o barrios pobres (Neufeld y Thisted, 1999; Filmus, 2003; Llomovate y Kaplan, 2005).

Estos y otros abordajes socio-culturales sobre las violencias escolares en el actual contexto de nuestro país coinciden en la delimitación de sus contornos principales (Antelo y Abramoswsky, 2000; Duschatzky, 1999; Duschatzky y Corea, 2002): 
(...) lo que la violencia como acto trae de diferente es que se sitúa fuera del lenguaje, diferenciándose así de los actos de transgresión a lo instituido donde la pretensión es disputar ideas e imaginarios (...). De este modo, lo que caracteriza la violencia actual en la escuela es la ausencia de nuevos modos de pensar y hacer frente a la "violencia", obturados por el discurso higiénico psico-didáctico tradicionalmente utilizado para dar cuenta del orden escolar. En este sentido, lo que el contexto reclama es escuchar no sólo "al otro" -como proponen las perspectivas más ligadas a la resolución de conflictos- sino "otra cosa" (Filmus, 2003, p. 25).

\section{Factores institucionales}

Otro núcleo sobre el cual se vienen desarrollando numerosas investigaciones sociales durante los últimos años pone su foco en las vinculaciones entre las violencias y los climas sociales escolares (CSE). Mediante dicha categoría se busca analizar las articulaciones entre a) la percepción que tienen los sujetos (docentes, directivos, preceptores, alumnos, padres) acerca de las relaciones interpersonales que establecen en el contexto escolar y b) el contexto o marco en el cual estas interacciones se dan (a nivel del aula o de toda la institución educativa). Como surge de diversos estudios contemporáneos, el CSE no es sólo una dimensión secundaria que afecta externamente las prácticas, los procesos de aprendizaje, la convivencia, la conflictividad y, en general, las experiencias sociales de sus agentes, sino que es, en sí misma, constitutiva de éstos (Cornejo y Redondo, 2001; Dupper y Meyer-Adams, 2002; Onetto, 2004; Abramovay, 2006; Kornblit, 2008; Di Leo, 2009).

En Francia, las investigaciones sobre las vinculaciones entre los CSE y las violencias cotidianas generaron una verdadera ruptura epistemológica con respecto a los abordajes tradicionales del problema. Impulsado por una demanda del Estado francés hacia el campo académico para tener un conocimiento más complejo de la temática, se inicia en 1994 un vasto programa de estudios empíricos que, por primera vez, buscan construir datos sobre los sentidos y experiencias de los diversos actores de la comunidad escolar frente a las problemáticas de la convivencia y las violencias. Se utilizan variadas herramientas metodológicas: "relatos de violencia", cuestionarios por correspondencia o directamente aplicados en escuelas, grupos de reflexión, entrevistas individuales, investigaciones sobre victimización, análisis secundario de datos estadísticos o de documentos administrativos, observaciones etnográficas, estudios de caso e investigación-acción por mediación sociológica (Debarbieux, 2001).

En esta misma línea, a partir de diversos trabajos de investigación social de alcance nacional desarrollados por nuestro equipo, comprobamos una efectiva asociación entre las condiciones institucionales y los niveles de violencia experimentados por distintos sujetos en la cotidianeidad escolar. A medida que 
empeora el CSE, se incrementan significativamente los episodios de violencia y lo inverso sucede cuando el CSE mejora (Kornblit, 2008). Esta asociación también fue hallada en estudios realizados por el Observatorio Argentino de Violencia en las Escuelas y en otras investigaciones realizadas recientemente a nivel nacional e internacional (Bertella, 2001; Abramovay, 2006; Míguez, 2008; Kaplan, 2009).

Según uno de los estudios desarrollados por nuestro equipo de investigación, entre las diversas dimensiones del CSE analizadas, la que tiene una mayor incidencia en las manifestaciones de violencias escolares es el grado de autoritarismo de los docentes -tal como es percibido por los estudiantes. Relaciones similares emergen alrededor de otros de los aspectos centrales de la relación docente-alumno -valorar del esfuerzo de los estudiantes y propiciar la comunicación y la integración-: a mayor presencia de estas dimensiones en las relaciones pedagógicas, aumenta la proporción de estudiantes menos vulnerables y menos protagonistas de situaciones de violencia en la escuela. Por ende, a partir de estos análisis surge que el vínculo docente-alumno se constituye en uno de los factores protectores más importante frente a las manifestaciones de violencia escolar (Kornblit, 2008).

En mi investigación doctoral -desarrollada entre 2005 y 2007 en dos escuelas secundarias públicas de la CABA-, a partir del análisis de las prácticas y discursos de los docentes, directivos y estudiantes -siguiendo los lineamientos generales de la teoría fundamentada (Strauss y Corbin, 2006)-, dialogando con diversas herramientas conceptuales y con el estado del arte, identifiqué la presencia, en ambas instituciones educativas, de dos tipos de CSE dominantes -desubjetivante e integracionista-normativo-, cuyas principales características son: ${ }^{3}$

\section{a) Clima social escolar desubjetivante:}

Las significaciones y vinculaciones de la mayoría de los docentes y directivos con los jóvenes se basan en la negación de sus capacidades de agencia y/o reflexividades, tanto desde posiciones discursivas naturalistas y/o psicologistas -falta de maduración-como desde su victimización social-objeto pasivo de las transformaciones económico-sociales y culturales (Chaves, 2005). Se negativizan sus prácticas, asociándolas fundamentalmente a las violencias, transgresiones y/o riesgos sociales -drogas, delitos, ITS, VIH/sida, etc. Sólo se les reconoce su capacidad estratégica para la generación de tácticas de impugnación de las normas/autoridades escolares, invisibilizando sus potencialidades críticas y/o instituyentes.

La percepción de esta profunda brecha entre el mundo de los adolescentes -no reflexivo, incivilizado, violento-y el de los adultos -reflexivo, civilizado-, genera en los docentes y directivos sensaciones de una creciente incapacidad para ejercer el rol para el cual fueron formados y, en general, de una 
profunda crisis de la autoridad escolar y de la institución educativa, las que a su vez profundizan sus sentimientos de malestar y estrés laboral. Asimismo, las consecuencias de estos fenómenos en la práctica pedagógica -desgano, dificultades para controlar las propias reacciones y establecer una comunicación con estudiantes y pares- retroalimentan en un círculo vicioso los procesos de desubjetivación tanto de sí mismos -objetos de las circunstancias- como de los otros.

En este tipo de CSE dominante se coloca a la crisis de la autoridad escolar como una de las principales causas y, simultáneamente, como una de las consecuencias más graves de las violencias escolares. Desde una posición discursiva normativo-disciplinar dominante, consideran que la crisis de la autoridad escolar se origina en el debilitamiento de sus principios de legitimidad clásicos -tradicional y/o racional-burocrático- (Weber, 1997). Ven a las propuestas y/o experiencias de dispositivos institucionales y/o pedagógicos destinados a gestionar la convivencia escolar de maneras no tradicionales -consejos de convivencia, consejos de aula, tutorías, talleres- como lentos y/o ineficientes, llegando a incluirlos entre los factores causales de la crisis de la autoridad y el aumento de episodios de violencias en la escuela. Por ende, propician un retorno a los dispositivos normativo-disciplinares tradicionales como la única vía para reconstruir la legitimidad y la eficacia de la autoridad escolar (Di Leo, 2009).

\section{b) Clima social escolar integracionista-normativo:}

Al igual que en el tipo de CSE anterior, los agentes escolares visibilizan como un grave problema los diversos tipos de violencias -físicas, psicológicas y simbólicas- presentes en la cotidianeidad de la escuela secundaria. Sin embargo, se diferencian en la identificación de las causas y, por lo tanto, en las posibles vías de revertir las mismas. Partiendo de formaciones discursivas de la patología social, del pánico moral y/o sociologistas, consideran que las violencias escolares se originan en las recientes transformaciones de las condiciones económico-sociales y/o políticas de nuestro país, que generaron una ampliación del área de vulnerabilidad y/o exclusión social, a las que los jóvenes/ adolescentes se encuentran especialmente expuestos (Chaves, 2005).

Estas nuevas condiciones estructurales ponen en crisis las funciones tradicionales de la escuela -lo que a su vez genera conflictos entre sus agentes-, interpelándola para modificar sus proyectos y arreglos institucionales en función del objetivo central de integrar a los jóvenes/adolescentes "a como dé lugar" a las instituciones de socialización clásicas -familia, escuela, trabajo(Reguillo, 2004). Dentro de dichas transformaciones, se considera que deben realizarse profundos cambios en los fundamentos del rol docente, desplazando sus tradicionales funciones normativo-disciplinares y poniendo en el centro a las dimensiones vocacionales de la profesión -compromiso, creatividad e iniciativa personales. 
En este otro tipo de CSE dominante, los docentes y directivos coinciden en el diagnóstico de la crisis de la autoridad escolar, aunque no la califican como un fenómeno negativo en sí mismo, sino que la ven también como una posibilidad de superar relaciones autoritarias generadas desde muchos docentes y/o directivos. En el mismo sentido que los citados cambios del rol docente, consideran que la autoridad escolar debería desplazar sus principios de legitimación tradicional y/o racional-burocrático, poniendo en el centro los aspectos personales de los agentes que favorezcan la reconstrucción de la confianza de los estudiantes en ellos y, por su intermedio, en las normas y rituales escolares -tipo de legitimidad carismática (Weber, 1997; Di Leo, 2009).

\section{Experiencias escolares}

Durante los últimos años, retomando, entre otras influencias teóricas, las propuestas de la sociología de la experiencia de François Dubet y Danilo Martuccelli (1998; 2000), diversas investigaciones desarrolladas en Argentina se centran en el análisis de las características y heterogeneidades de las experiencias escolares. Si bien no todos estos trabajos se centran en la problemática de las violencias, en su mayoría suministran valiosos datos para analizar las diversas tácticas y estrategias desplegadas por los jóvenes en los procesos de construcción de sus subjetividades en contextos escolares. Por ende, a partir de este tipo de abordajes es posible reconstruir la trama compleja de las violencias escolares articulando sus dimensiones políticas, histórico-sociales, institucionales e individuales desde la perspectiva de los actores, buscando superar los dualismos objetivismo / subjetivismo, estructura / sujeto.

En esta línea, según los trabajos de Silvia Duschatzky (1999), Cristina Corea (Duschatzky y Corea; 2002) y Gabriel Noel (2009) las violencias vividas y percibidas por los jóvenes de sectores populares se encuentran marcadas por la corporalidad. Mientras que las autoras vinculan esta dimensión al incremento en el contexto barrial del consumo de drogas y otras manifestaciones de violencia en sentido estricto -tales como agresiones físicas-, Noel señala que, si bien estas formas de vinculación constituyen una realidad innegable, no tienen la omnipresencia ni la homogeneidad que suelen atribuirle los agentes escolares. Esto últimos asignan, en gran medida, la calificación de violentas a las interacciones entre alumnos debido a una diferencia sistemática de expectativas relacionadas con los usos correctos del cuerpo. Dicha distancia estaría marcada, por un lado, por la corporalidad canónica de los agentes del sistema escolar socializados en sectores medios y, por otro lado, por las pretensiones civilizatorias de la escuela.

De esta manera, por ejemplo, uno de los principales núcleos conflictivos entre estudiantes y agentes escolares tiene que ver con las cuestiones indumentarias. Si bien las escuelas ubicadas en barrios de sectores populares suelen ser relativamente laxas en la aplicación de criterios de vestimenta 
-no prescriben, por ejemplo, el uso del guardapolvo-, existen determinadas elecciones valoradas en los estilos expresivos de los jóvenes que suscitan en muchos casos instantáneos llamados de atención por parte de los agentes del sistema escolar: el uso de la gorra con visera y de la capucha de los buzos. Esto pone de manifiesto uno de los dilemas centrales en las instituciones educativas: la oposición entre la continuidad y la discontinuidad entre la escuela y el afuera. Por un lado, se ubican los agentes pragmáticos, que defienden la necesidad de adaptar la escuela y sus demandas a las particularidades culturales de las poblaciones que recibe, a los que se les enfrentan los normativos, que señalan la importancia de marcar una clara distinción entre un afuera y un adentro institucional, que prepararía a los estudiantes para el acceso futuro a entornos socio-culturalmente diferenciados de los de sus barrios de origen (Noel, 2009).

Uno de los ejes analíticos centrales en las investigaciones de Gabriel Kessler (2002) en instituciones educativas es la relación ambigua de los sujetos con las reglas, la disciplina y las normas de convivencia. Más allá de las profundas diferencias detectadas en las experiencias escolares de jóvenes provenientes de distintos estratos socioeconómicos, el autor identifica como una constante la coexistencia de reglas que en teoría son válidas, prohibiendo determinadas prácticas, pero que en la realidad cotidiana no tienen el poder de inhibir su ocurrencia. Si bien no hay un cuestionamiento explícito a las normas en general y a su valor en el proceso de formación personal, en la práctica emerge la sensación de que todo es negociable, toda regla es franqueable. Los límites a esta maleabilidad no dependen tanto del sector social del que provenga los alumnos, sino del tipo de regulación interna y contrato de convivencia que se establezca en cada institución.

En el mismo sentido de los análisis de Dubet y Martuccelli (1998), Kessler (2002) identifica una gran variedad de estrategias desplegadas por los estudiantes para negociar con las normas de las instituciones escolares. Los resultados de estos procesos de negociación constante estarán en gran medida condicionados por los niveles de acumulación de diversos tipos de capital, fundamentalmente simbólico, con los que cuenten los agentes educativos. Mientras que en las escuelas a las que asisten los sectores altos este juego parece reforzar las dinámicas meritocráticas familiares, a medida que se desciende en la escala social se va acentuando la sensación de precariedad en las pautas de convivencia escolar, llegando en los sectores marginales a una fuerte naturalización de episodios cotidianos de violencia en sentido estricto (amenazas, golpes, robos). En otro de sus trabajos, el sociólogo define a la inserción educativa de jóvenes que participaron en delitos contra la propiedad con utilización de la violencia como escolaridad de baja intensidad: se produce un desenganche entre sus procesos de subjetivación -constituidos desde otros espacios de socialización-y la escuela (Kessler, 2004). 
En esta misma línea, Noel (2009) señala que la convivencia en escuelas a las que asisten jóvenes de sectores populares se encuentra marcada por una conflictividad permanente que, en muy raras ocasiones, puede resolverse mediante mecanismos institucionales. A partir del análisis de estas experiencias, Duschatzky y Corea (2002) formulan la hipótesis del declive de las instituciones familiares y escolares en tiempos de fragmentación social. Las mismas pierden su poder de subjetivación en relación a las experiencias juveniles, en las que adquieren mayor protagonismo los ritos, las creencias, el "choreo" (robo), el consumo, el "faneo" (drogarse), etc. De esta manera, la sociabilidad entre pares se configura como alternativa frente a la ineficacia simbólica de las instituciones tradicionales (familia y escuela).

A partir de mi investigación doctoral, en la que utilicé diversas técnicas de investigación social de tipo cualitativo -observaciones participantes y no participantes, entrevistas semiestructuradas, grupos focales y diarios personales (en la que los estudiantes narran diversos momentos de sus biografías escolares)-, identifiqué que los jóvenes significan a la escuela como un escenario fragmentado, con escasa capacidad para habilitar espacios para participar, expresar sus inquietudes y/o intervenir previniendo o ayudando a solucionar problemas vinculados a sus experiencias cotidianas. Sin embargo, simultáneamente, la institución educativa es vivida por ellos como un espacio en el que cotidianamente despliegan sus luchas por el reconocimiento (Honneth, 1997). ${ }^{4}$ En este marco, otorgan especial importancia a las miradas de los otros, reaccionando -física y/o discursivamente- al percibir el desprecio y/o la discriminación de sus pares. Asimismo, consideran especialmente violentas las prácticas discriminatorias, los abusos de poder y/o las injusticias de los adultos en el contexto escolar (Di Leo, 2009).

En diálogo con las investigaciones de Kessler (2002) y Noel (2009), identifiqué dos tipos de tácticas de impugnación de la autoridad presentes en las relaciones cotidianas de los estudiantes con los agentes escolares: a) personal y b) jurídico-moral. Mientras el primer tipo se dirige hacia dimensiones personales de algunos docentes y/o directivos consideradas contradictorias con el ejercicio de su rol, la impugnación jurídico-moral centra la crítica en determinadas normas y/o rituales escolares por considerarlos injustos y/o autoritarios. Esta última táctica, con una mayor presencia en los discursos y prácticas juveniles, manifiesta, por un lado, una forma de resistencia frente a los intentos de los agentes -principalmente desde el CSE desubjetivante- por reconstruir la legitimidad tradicional y/o racional-legal de la autoridad escolar, soslayando su carácter político y, por lo tanto, abierto, dialógico e indeterminado. Sin embargo, la dimensión negativa de este tipo de tácticas se articula, en muchos casos, con un momento positivo: a partir de las mismas los jóvenes luchan por ser reconocidos como seres genéricos, sujetos jurídico-morales plenos, buscando construir puentes entre sus experiencias y valores personales y los sistemas expertos representados por la institución escolar y sus agentes (Di Leo, 2009). ${ }^{5}$ 
A partir del análisis de las narraciones biográficas de los estudiantes encontramos que las relaciones de confianza -tanto con sus pares como, especialmente, con los adultos- ocupan un lugar central en los trabajos de construcción de sus experiencias escolares en dos procesos interrelacionados: a) en la reducción de la creciente complejidad sistémica -propia de la actual etapa de la segunda modernidad-, contribuyendo al mantenimiento de su seguridad ontológica y b) en la construcción de núcleos de articulación entre sus experiencias vitales cotidianas y los sistemas expertos -principalmente a partir de vínculos de confianza con algunos agentes escolares- (Luhmann, 1996; Giddens, 1991; Di Leo, 2009).

Asociado a este segundo proceso, para la mayoría de los jóvenes la autoridad escolar ya no puede fundarse en principios pre-políticos, sino que debe legitimarse cotidianamente a partir de relaciones de confianza, argumentación y diálogo intersubjetivos, sin suturar sus sentidos a partir de un único discurso, saber y/o imaginario efectivo institucionalizado. De esta manera, en la línea de los análisis de Beatriz Greco (2007), podemos reconocer en estas tácticas juveniles la potencialidad para la creación de un tipo de autoridad escolar que no busque fundamentarse exclusivamente en los tipos de legitimidad tradicional, racional-burocrático y/o carismático, sino que se vaya construyendo a partir de relaciones de confianza instituyente:

(...) es la manera en que podemos nombrar esta posición de autoridad que se autoriza a sí misma al liberar al otro del afán de control y la sospecha permanente. Confianza que tiene la potencia de anticipar lo que va a ir ocurriendo en la relación porque hace que el otro se sienta reconocido y pueda responder desde imágenes valorizadas de sí mismo, en el marco de una relación que sostiene un trabajo por hacer. Confianza que no es garantía absoluta, sino riesgo de que muchas idas y vueltas ocurran en esa relación (Greco, 2007, p. 48).

\section{A modo de cierre y apertura: tensiones y potencialidades de las experiencias escolares}

Las experiencias escolares juveniles se encuentran marcadas por la fragmentación, las escisiones y los conflictos. Por un lado, se repiten cotidianamente un conjunto de rituales de orden y control disciplinario que, en un lugar de construir un espacio simbólico de reconocimiento, profundizan las sensaciones de falta de sentido y de distanciamiento de los jóvenes con respecto al mundo de los adultos y a la institución educativa. Retomando los análisis de Dubet y Martuccelli (1998, 2000; Dubet, 2006), es posible afirmar que estas experiencias juveniles no son sólo síntomas de una crisis de las instituciones encargadas de la socialización (familia, escuela), una prueba de su incapacidad de adaptación a nuevas situaciones, sino que se enmarcan en un movimiento profundo de desinstitucionalización: una nueva modalidad de vinculación entre valores, 
normas e individuos; es decir, una nueva forma de socialización. Los valores y las normas ya no pueden ser representados como entidades trascendentales, ahistóricas, por encima de las experiencias individuales. En cambio, aparecen como producciones sociales, conjuntos compuestos por principios de integración múltiples y a menudo contradictorios. Por consiguiente, este movimiento genera una creciente separación de dos procesos que la sociología clásica confundió: la socialización y la subjetivación.

Las subculturas, los cuerpos y las experiencias juveniles, durante mucho tiempo mantenidas fuera de los muros de la escuela, irrumpieron en la misma, con sus modelos, conflictos y preocupaciones. Las nuevas pautas de socialización juvenil hacen evidente, por contraposición, la pérdida de importancia de la cultura escolar clásica. De igual modo, la fragmentación, tanto del sistema educativo en su conjunto como al interior de las instituciones escolares, disminuyen sus potencialidades para contrarrestar los procesos de descivilización generados por décadas de exclusión económico-social: las heterocoacciones, el bajo control de las emociones, la discriminación, las agresiones, la inestabilidad emocional, entre otros, se convierten en síntomas de la expansión de un individualismo negativo.

En este marco, las manifestaciones de violencia antiescolar en las instituciones educativas de nivel secundario -denunciadas cotidianamente por las y los docentes y directivos- pueden leerse, al menos parcialmente, como reacciones frente a experiencias de injusticia, autoritarismo, faltas de respeto y/o desprecio generadas desde el mundo adulto -especialmente en los CSE desubjetivante e integracionista-normativo. Por lo tanto, en lugar de identificar estas prácticas discursivas juveniles únicamente cómo síntomas de la crisis y/o ausencia de la autoridad escolar, también pueden interpretarse como expresiones de las luchas de los jóvenes por ser reconocidos como seres genéricos, como sujetos en los planos jurídico-moral y social que, a pesar de su crisis, siguen estando representados por la escuela (Di Leo, 2009).

Frente a los citados CSE hoy dominantes en las instituciones educativas, las relaciones de confianza -con los pares y, especialmente, con los adultos-, ocupan un lugar central en las experiencias escolares de los jóvenes. Las mismas se presentan en sus relatos como un proceso en constante negociación, organizado por una serie de símbolos que van siendo resignificados y/o actualizados a partir de rituales cotidianos de sociabilidad. Los estudiantes reconocen y valoran a aquellos docentes y directivos que en sus interacciones cotidianas presentan una apertura para la escucha, el diálogo y, en general, el vínculo intersubjetivo con los otros (Di Leo, 2009).

A nuestro entender, este tipo de relaciones de confianza instituyente puede servir de base para la emergencia de autoridades, rituales y normas escolares que no busquen legitimarse exclusivamente en la tradición, la racionalidad burocrática y/o el carisma, si no que se fundamenten en los trabajos cotidianos de 
escucha, apertura y reconocimiento de los otros. A partir del diálogo entre las experiencias juveniles y los saberes expertos representados por la institución escolar y sus agentes, se abren nuevas posibilidades para la relegitimación de la escuela en el actual contexto de nuestras sociedades democráticas y para su (re)construcción como un espacio público, en el sentido propuesto por Hannah Arendt: ámbito privilegiado para el ejercicio de la libertad de los sujetos -que requiere la visibilidad por y con el otro-, ya que constituye el territorio privilegiado de producción simultánea de intersubjetividades y singularidades (Arendt, 1993).

\section{Notas bibliográficas}

(1) Dichos trabajos de investigación se enmarcaron en los proyectos: a) PICT $04 \mathrm{~N}^{\circ} 13.284$, financiado por la Agencia Nacional de Investigaciones Científicas y Técnicas; b) UBACyT S071, financiado por la Universidad de Buenos Aires y c) "Clima social y niveles de violencia en escuelas secundarias de todo el país", financiado por la Dirección Nacional de Información y Evaluación de la Calidad Educativa (DINIECE) del Ministerio de Educación de la Nación.

(2) Según Pierre Bourdieu, las instituciones socializadoras modernas -entre las cuales la escuela ocupa un lugar central- producen y reproducen un conjunto de categorías de percepción, motivaciones, intereses, prácticas impensadas, que son incorporadas en los cuerpos y las conciencias de los agentes como una segunda naturaleza -habitus. La participación de los agentes en los diversos campos que componen el espacio social depende de la incorporación (más allá de la conciencia y la voluntad) de una doxa, conjunto de reglas prácticas, de axiomas que no se cuestionan y que, como las categorías de percepción y valoración del resto de los capitales que están en juego en cada campo, son reproducidas a través el ejercicio de la violencia simbólica (Bourdieu, 2007).

(3) Si bien, para no exceder los objetivos del artículo, aquí nos centramos en los dos tipos de clima social escolar (CSE) dominantes, a partir de mi investigación identifiqué la presencia de un tercer tipo de CSE -ético-subjetivante-, que se manifestaba en las instituciones educativas de manera fragmentada y subordinada con respecto a los otros dos (Di Leo, 2009).

(4) Según Axel Honneth, para el análisis de las diversas modalidades de reconocimiento en relación a los cuales se van constituyendo las subjetividades es necesario darles cuerpo, concretizarlas, abordándolas en relación a las formas de menosprecio o de negación de reconocimiento que movilizan a los individuos a luchar por ser reconocidos en los heterogéneos contextos intersubjetivos que atraviesan en sus vidas cotidianas. De esta manera, identifica tres grandes modelos de reconocimiento: a) afectivo, b) jurídico-moral y c) ético-social (Honneth, 1997).

(5) Entre los principales mecanismos que participan de la dialéctica de desanclaje-rearticulación entre las instituciones sociales y las identidades personales en la segunda modernidad, Anthony Giddens analiza dos grandes tipos de sistemas abstractos: a) medios simbólicos -medios de cambio en una pluralidad de contextos (por ejemplo, la moneda)- y b) sistemas expertos -saberes legitimados institucionalmente. A partir del influjo de los mismos, de manera creciente las relaciones, autoridades y normas sociales son susceptibles de permanentes revisiones crónicas a la luz de las nuevas informaciones y/o conocimientos que circulan globalmente (Giddens, 1991). 


\section{Referencias bibliográficas}

- Abramovay, M. \& Das Graças Rua, M (2002). Violência nas escolas. Brasilia: UNESCO.

- Abramovay, M. (coord.) (2006). Cotidiano das escolas: entre violências. Brasilia: UNESCO, Observatório de violências nas escolas, Ministerio da Educaçao.

- Antelo, E. \& Abramoswsky, A. (2000). El renegar de la escuela: desinterés, apatía, aburrimiento, violencia e indisciplina. Rosario: Homo Sapiens.

- $\quad$ Arendt, H. (1993). La condición humana. Buenos Aires: Paidós.

- Bertella, M. L. (2001). La convivencia en escuelas secundarias de contextos desfavorables. Revista del IICE, 18, 59-71.

- Bourdieu, P. \& Passeron, J.-C. (1981). La reproducción. Elementos para una teoría del sistema de enseñanza. Barcelona: Laia.

- $\quad$ Bourdieu, P. (2007). El sentido práctico. Buenos Aires: Siglo XXI.

- $\quad$ Castel, R. (1997). La metamorfosis de la cuestión social. Una crónica del asalariado. Buenos Aires: Paidós.

- $\quad$ Charlot, B. \& Emin, J. C. (Coord.) (1997). Violences á l'école. État des savoirs. Paris: Armand Colin.

- Charlot, B. (2002). A violência na escola, como os sociólogos franceses abordam essa questão. Sociologías, 8, 432-443.

- Charlot, B. (2006). Prefácio. En M. Abramovay (Coord.). Cotidiano das escolas, entre violências (pp. 17-25). Brasilia: UNESCO, Observatório de violências nas escolas, Ministerio da Educaçao.

- $\quad$ Chaves, M. (2005). Juventud negada y negativizada: representaciones y formaciones discursivas vigentes en la Argentina contemporánea. Última Década, 23, 9-32.

- $\quad$ Corea, C. \& Lewkowicz, I. (2004). Pedagogía del aburrido. Escuelas destituidas, familias perplejas. Buenos Aires: Paidós.

- $\quad$ Cornejo, R. \& Redondo, J. (2001). El clima escolar percibido por los alumnos de enseñanza media. Una investigación en algunos liceos de la Región Metropolitana. Última década, 15, 11-52.

- Debarbieux, E. (1996). La Violence en Milieu Scolaire 1. Etat des lieux. Paris: ESF.

- Debarbieux, E. (2001). A violência na escola francesa, 30 anos de construção social do objeto (1967-1997). Educação e Pesquisa, 27(1), 163-193.

- Di Leo, P. F. (2009). Subjetivación, violencias y climas sociales escolares. Un análisis de sus vinculaciones con experiencias de promoción de la salud en escuelas secundarias públicas de la Ciudad Autónoma de Buenos Aires. Tesis Doctoral en Ciencias Sociales, Universidad de Buenos Aires, no publicada.

- Dubet, F. \& Martuccelli, D. (1998). En la escuela. Sociología de la experiencia escolar. Buenos Aires: Losada.

- $\quad$ Dubet, F. \& Martuccelli, D. (2000). ¿En qué sociedad vivimos? Buenos Aires: Losada.

- Dubet, F. (2006). El declive de la institución. Profesiones, sujetos e individuos en la modernidad. Barcelona: Gedisa.

- Dupper D. \& Meyer-Adams N. (2002). Low level violence. A neglected aspect of school culture. Urban Education, 5 (37), 350-364.

- $\quad$ Durkheim, E. (1972). La educación moral. Buenos Aires: Shapire.

- Durkheim, E. (1998). Educación y pedagogía. Ensayos y controversias. Buenos Aires: Losada.

- Duschatzky, S. \& Corea, C. (2002). Chicos en banda. Los caminos de la subjetividad en el declive de las instituciones. Buenos Aires: Paidós. 
- Duschatzky, S. (1999). La escuela como frontera. Reflexiones sobre la experiencia escolar de jóvenes de sectores populares. Buenos Aires: Paidós.

- Filmus, D. \& Miranda, A. (1999). América Latina y Argentina en los '90, más educación, menos trabajo = más desigualdad. En D. Filmus (Comp.). Los noventa. Política, sociedad y cultura en América Latina y Argentina de fin de siglo (pp.111-150). Buenos Aires: EUDEBA.

- $\quad$ Filmus, D. (2003). Enfrentando la violencia en las escuelas, Un informe de Argentina. En VVAA. Violência na Escola, América Latina y Caribe (pp. 15-85). Brasilia: UNESCO.

- $\quad$ Freire, P. (1997). Pedagogía del oprimido. Madrid: Siglo XXI.

- Gentili, P. (1994). Proyecto neoconservador y crisis educativa. Buenos Aires: CEAL.

- Giddens, A. (1991). Modernity and Self-Identity. Self and Society in the Late Modern Age. Oxford: Polity.

- Greco, M. B. (2007). La autoridad (pedagógica), en cuestión. Una crítica al concepto de autoridad en tiempos de transformación. Rosario: Homo Sapiens.

- Honneth, A. (1997). La lucha por el reconocimiento. Por una gramática moral de los conflictos sociales. Barcelona: Crítica.

- Kaplan, C. V. (Dir.) (2006). Violencias en plural. Sociología de las violencias en las escuelas. Buenos Aires: Miño y Dávila.

- Kaplan, C. V. (Dir.) (2009). Violencia escolar bajo sospecha. Buenos Aires: Miño y Dávila.

- Kessler, G. (2002). La experiencia social fragmentada. Estudiantes y docentes en la escuela secundaria en Buenos Aires. Buenos Aires: IIPE-UNESCO.

- Kessler, G. (2004). Sociología del delito amateur. Buenos Aires: Paidós.

- Kornblit, A. L. (Coord.) (2008). Violencia escolar y climas sociales. Buenos Aires: Biblos.

- Llomovate, S. \& Kaplan, C. (Coords.) (2005). Desigualdad educativa. La naturaleza como pretexto. Buenos Aires: Noveduc.

- Luhmann, N. (1996). Confianza. México: Anthropos.

- Míguez, D. (2000). Lo privado en lo público durante la modernidad radicalizada. Las relaciones de autoridad y sus dilemas en las organizaciones socializadoras argentinas. Espacios en Blanco. Revista de Educación, 10, 49-68.

- Míguez, D. (Comp.) (2008). Violencias y conflictos en las escuelas. Buenos Aires: Paidós.

- $\quad$ Neufeld, M. R. \& Thisted, J. A. (1999). De eso no se habla. Los usos de la diversidad sociocultural en la escuela. Buenos Aires: EUDEBA.

- Noel, G. (2009). La Conflictividad Cotidiana en el Escenario Escolar. Una Perspectiva Etnográfica. Buenos Aires: UNSAM.

- Onetto, F. (2004). Climas educativos y pronósticos de violencia. Condiciones institucionales de la convivencia escolar. Buenos Aires: NOVEDUC.

- $\quad$ Payet, J. P. (1997). "Violence á l'école, Les coulisses de procés”. En B. Charlot y J. C. Emin (Coord.). Violences á l'école. État des savoirs. Paris: Armand Colin.

- Reguillo, R. (2004). La performatividad de las culturas juveniles. En Estudios de Juventud, 64, 49-56.

- $\quad$ Strauss, A. \& Corbin, J. (2006). Bases de la investigación cualitativa. Técnicas y procedimientos para desarrollar la teoría fundamentada. Bogotá: CONTUS, Universidad de Antioquía.

- Tedesco, J. C. (2008). ¿Son posibles las políticas de subjetividad? En E. Tenti Fanfani (Comp.). Nuevos temas en la agenda de política educativa (pp. 53-64). Buenos Aires: IIPE, UNESCO, Siglo XXI. 
- Tenti Fanfani, E. (1999). Civilización y descivilización. Norbert Elias y Pierre Bourdieu, intérpretes de la cuestión social contemporánea. Sociedad, 14, 7-28.

- Tenti Fanfani, E. (2007). Algunas consecuencias de la escolarización masiva. TodaVÍA, 17. Disponible en: http://www.revistatodavia.com.ar/todavia17/notas/fanfani/txtfanfani.html

- Tiramonti, G. \& Montes, N. (Comp.) (2008). La escuela media en debate. Problemas actuales y perspectivas desde la investigación. Buenos Aires: FLACSO, Manantial.

- Weber, M. (1997). Economía y sociedad. México D.F.: FCE. 